Kragujevac Journal of Mathematics

Volume 46(1) (2022), Pages 139-148.

\title{
A NOTE ON COMPARISON OF ANNULI CONTAINING ALL THE ZEROS OF A POLYNOMIAL
}

\author{
SUNIL HANS ${ }^{1}$, AMIT TOMAR ${ }^{1}$, AND JIANHENG CHEN ${ }^{2}$
}

\begin{abstract}
If $P(z)$ is a polynomial of degree $n$, then for a subclass of polynomials, Dalal and Govil [7] compared the bounds, containing all the zeros, for two different results with two different real sequences $\lambda_{k}>0, \sum_{k=1}^{n} \lambda_{k}=1$. In this paper, we prove a more general result, by which one can compare the bounds of two different results with the same sequence of real or complex $\lambda_{k}, \sum_{k=0}^{n}\left|\lambda_{k}\right| \leq 1$. A variety of other results have been extended in this direction, which in particular include several known extensions and generalizations of a classical result of Cauchy [4], from this result by a fairly uniform manner.
\end{abstract}

\section{INTRODUCTION}

Properties of polynomials and especially the location of zeros is an old subject and it has been studied since the time of Gauss and Cauchy, as it finds important application in many areas of applied mathematics. Quite a few results giving the bound for some and all the zeros of a polynomial in terms of its coefficients can be found in [13-15].

Gauss was the earliest contributor to the study of location of zeros of a polynomial, who proved that a $n^{\text {th }}$ degree polynomial

$$
P(z):=a_{n} z^{n}+a_{n-1} z^{n-1}+\cdots+a_{1} z+a_{0},
$$

with all real $a_{k}$, has no zeros outside the circle $|z|=R$, where

$$
R=\max _{1 \leq k \leq n}\left(n \sqrt{2}\left|a_{k}\right|\right)^{1 / k} .
$$

Cauchy [4] improved the above result of Gauss by proving the following result.

Key words and phrases. Polynomial, zeros, Fibonacci's numbers.

2010 Mathematics Subject Classification. Primary: 30C15. Secondary: 26C10.

DOI 10.46793/KgJMat2201.139H

Received: November 16, 2018.

Accepted: September 11, 2019. 
Theorem 1.1. Let $P(z)=\sum_{k=0}^{n} a_{k} z^{k}$ be a complex polynomial of degree $n$. Then all the zeros of $P(z)$ lie in the disc

$$
\{z:|z| \leq r\} \subset\{z:|z|<1+M\}
$$

where

$$
M:=\max _{0 \leq k \leq n-1} \frac{\left|a_{k}\right|}{\left|a_{n}\right|}
$$

and $r$ is the unique positive real root of the real coefficients equation

$$
\left|a_{n}\right| z^{n}-\left|a_{n-1}\right| z^{n-1}-\left|a_{n-2}\right| z^{n-2}-\cdots-\left|a_{1}\right| z-\left|a_{0}\right|=0,
$$

when $P(z)$ is not a monomial, and as zero otherwise.

On applying the above theorem to the polynomial $Q(z)=z^{n} P(1 / z)$ and combining it with the above theorem, one can easily get the following theorem.

Theorem 1.2 (Cauchy). All the zeros of a $n^{\text {th }}$ degree polynomial $P(z)=\sum_{k=0}^{n} a_{k} z^{k}$ lie in the annulus $r_{1} \leq|z| \leq r_{2}$, where $r_{1}$ is the unique positive real root of the equation

$$
\left|a_{n}\right| z^{n}+\left|a_{n-1}\right| z^{n-1}+\cdots+\left|a_{1}\right| z-\left|a_{0}\right|=0,
$$

and $r_{2}$ is the unique positive real root of the equation

$$
\left|a_{n}\right| z^{n}-\left|a_{n-1}\right| z^{n-1}-\cdots-\left|a_{1}\right| z-\left|a_{0}\right|=0,
$$

when $P(z)$ is not a monomial, and as zero otherwise.

The above result of Cauchy has been sharpened among others by Joyal et al. [11], Datt and Govil [8], Affane-Aji et al. [1], and Sun and Hsieh [16].

Diaz-Barrero [9] proved the following result, which provides an annulus containing all the zeros of a polynomial by involving the Fibonacci numbers and binomial coefficients.

Theorem 1.3. Let $P(z)=\sum_{k=0}^{n} a_{k} z^{k}, a_{k} \neq 0,1 \leq k \leq n$, be a non-constant polynomial with complex coefficients. Then all its zeros lie in the annulus $\mathcal{C}=$ $\left\{z: r_{1} \leq|z| \leq r_{2}\right\}$, where

$$
r_{1}=\frac{3}{2} \min _{1 \leq k \leq n}\left(\frac{2^{n} F_{k} C(n, k)}{F_{4 n}}\left|\frac{a_{0}}{a_{k}}\right|\right)^{1 / k}
$$

and

$$
r_{2}=\frac{2}{3} \max _{1 \leq k \leq n}\left(\frac{F_{4 n}}{2^{n} F_{k} C(n, k)}\left|\frac{a_{n-k}}{a_{n}}\right|\right)^{1 / k} .
$$

Here $C(n, k)$ is the binomial coefficient and $F_{k}$ is the $k^{\text {th }}$ Fibonacci number.

Kim [12] used the identity $\sum_{k=0}^{n} C(n, k)=2^{n}$ to prove the following result. 
Theorem 1.4. Let $P(z)=\sum_{k=0}^{n} a_{k} z^{k}, a_{k} \neq 0,1 \leq k \leq n$, be a non-constant polynomial with complex coefficients. Then all the zeros of $P(z)$ lie in the annulus $\mathcal{C}=\left\{z: r_{1} \leq|z| \leq r_{2}\right\}$, where

$$
r_{1}=\min _{1 \leq k \leq n}\left(\frac{C(n, k)}{2^{n}-1}\left|\frac{a_{0}}{a_{k}}\right|\right)^{1 / k}
$$

and

$$
r_{2}=\max _{1 \leq k \leq n}\left(\frac{2^{n}-1}{C(n, k)}\left|\frac{a_{n-k}}{a_{n}}\right|\right)^{1 / k} .
$$

Here $C(n, k)$ is the binomial coefficient.

There are many results, including the above Theorems 1.3 and 1.4, available in this direction (for example, see $[3,5,10]$ ).

Aziz and Qayoom [2] (see also [5]) proved the following theorem which generalizes all the above results.

Theorem 1.5. Let $\lambda_{1}, \lambda_{2}, \ldots, \lambda_{n}$ be any set of $n$ real or complex numbers such that $\sum_{k=1}^{n}\left|\lambda_{k}\right| \leq 1$, and let $P(z)=\sum_{k=0}^{n} a_{k} z^{k}, a_{k} \neq 0,1 \leq k \leq n$, be a non-constant polynomial with complex coefficients. Then all the zeros of $P(z)$ lie in the annulus $\mathcal{C}=\left\{z: r_{1} \leq|z| \leq r_{2}\right\}$, where

$$
r_{1}=\min _{1 \leq k \leq n}\left|\lambda_{k} \frac{a_{0}}{a_{k}}\right|^{1 / k}
$$

and

$$
r_{2}=\max _{1 \leq k \leq n}\left|\frac{1}{\lambda_{k}} \frac{a_{n-k}}{a_{n}}\right|^{1 / k} .
$$

Theorem 1.5 can generate infinitely many results, including Theorem 1.2 to 1.4 , giving an annulus containing all the zeros of a polynomial, and over the years, mathematicians were comparing the bounds with the existing bounds in the literature by giving some examples. Dalal and Govil [6] have shown that these bounds cannot, in general, be compared, implying that every result obtained can be useful. More recently, Dalal and Govil [7] successfully compared the bounds of two different results with two different real sequences $\lambda_{k}>0, \sum_{k=0}^{n} \lambda_{k}=1$ for a subclass of polynomials.

The main aim of this paper is to prove the following more general results (Theorem 2.1, 2.2) of Section 2 using well known Hölder Inequality

$$
\sum_{k=1}^{n} \alpha_{k} \beta_{k} \leq\left(\sum_{k=1}^{n} \alpha_{k}^{q}\right)^{1 / q}\left(\sum_{k=1}^{n} \beta_{k}^{p}\right)^{1 / p},
$$

where $\alpha_{k}>0, \beta_{k}>0$ for all $1 \leq k \leq n$, and $p>1, q>1$, with $1 / p+1 / q=1$, by which one can easily compare the bounds of two different results with the same sequence of real or complex $\lambda_{k}, \sum_{k=0}^{n}\left|\lambda_{k}\right| \leq 1$. Our results not only extend Theorem 1.5 but also 
improve it. Besides that theorems like Theorem 1.3, 1.4 and other similar to them also extended and shown as corollaries of Theorem 2.1 in the Table 1.

As above mentioned, the annuli obtained by Theorem 2.1 of Section 2 and Theorem 1.5 cannot, in general, be compared, however under some condition by MATLAB, we construct example of polynomial, for which the bounds obtained by our Theorem 2.1 and 2.2 are considerably sharper than the bounds obtained by Theorem 1.5. This has been done in Section 4.

\section{Main Results}

Theorem 2.1. Let $\lambda_{1}, \lambda_{2}, \ldots, \lambda_{n}$ be any set of $n$ real or complex numbers such that $\sum_{k=1}^{n}\left|\lambda_{k}\right| \leq 1$. For $p$ and $q$ such that $p>1, q>1, \frac{1}{p}+\frac{1}{q}=1$, the non-constant polynomial $P(z)=\sum_{k=0}^{n} a_{k} z^{k}, a_{k} \neq 0,1 \leq k \leq n$, with complex coefficients, has all its zeros lie in the annulus $\mathcal{C}=\left\{z: r_{1}^{\prime} \leq|z| \leq r_{2}^{\prime}\right\}$, where

$$
r_{1}^{\prime}=\min _{1 \leq k \leq n}\left|\frac{\lambda_{k}^{1 / p}}{n^{1 / q}} \frac{a_{0}}{a_{k}}\right|^{1 / k}
$$

and

$$
r_{2}^{\prime}=\max _{1 \leq k \leq n}\left|\frac{n^{1 / q}}{\lambda_{k}^{1 / p}} \frac{a_{n-k}}{a_{n}}\right|^{1 / k} .
$$

Remark 2.1. If for all $1 \leq k \leq n$, we take $\left|\lambda_{k}\right|=1 / n$, then by using $1 / p+1 / q=1$, we have from Theorem 2.1

$$
r_{1}^{\prime}=\min _{1 \leq k \leq n}\left|\frac{1}{n^{1 / p} n^{1 / q}} \frac{a_{0}}{a_{k}}\right|^{1 / k}=\min _{1 \leq k \leq n}\left|\frac{1}{n} \frac{a_{0}}{a_{k}}\right|^{1 / k}
$$

and

$$
r_{2}^{\prime}=\max _{1 \leq k \leq n}\left|n \frac{a_{n-k}}{a_{n}}\right|^{1 / k}
$$

which is similar to the bounds of Theorem 1.5 with $\left|\lambda_{k}\right|=1 / n, 1 \leq k \leq n$, but by Theorem 2.8 of Dalal and Govil [7], it is easy to see that if $\left|\lambda_{k}\right|<1 / n, 1 \leq k \leq n$, then Theorem 2.1 always gives a better bound than Theorem 1.5. Although, we are not able to prove analytically that whether any condition required over the coefficients of a polynomial or not, but with the help of MATLAB, we construct 1 million random polynomials, without any condition on coefficients, with different degrees, random $\lambda_{k}$, with $\left|\lambda_{k}\right|<1 / n$, and random $p>1, q>1$, with $1 / p+1 / q=1$, and that shows the bounds of our Theorem 2.1 is always better than Theorem 1.5. For reference, a result of a $30^{\text {th }}$ degree polynomial has been shown in Figure 2.1 below.

Remark 2.2. Let $r$ be the positive root of the equation

$$
z^{n p}\left|a_{n}\right|^{p}-z^{(n-1) p} n^{p / q}\left|a_{n-1}\right|^{p}-\cdots-z^{p} n^{p / q}\left|a_{1}\right|^{p}-n^{p / q}\left|a_{0}\right|^{p}=0 .
$$



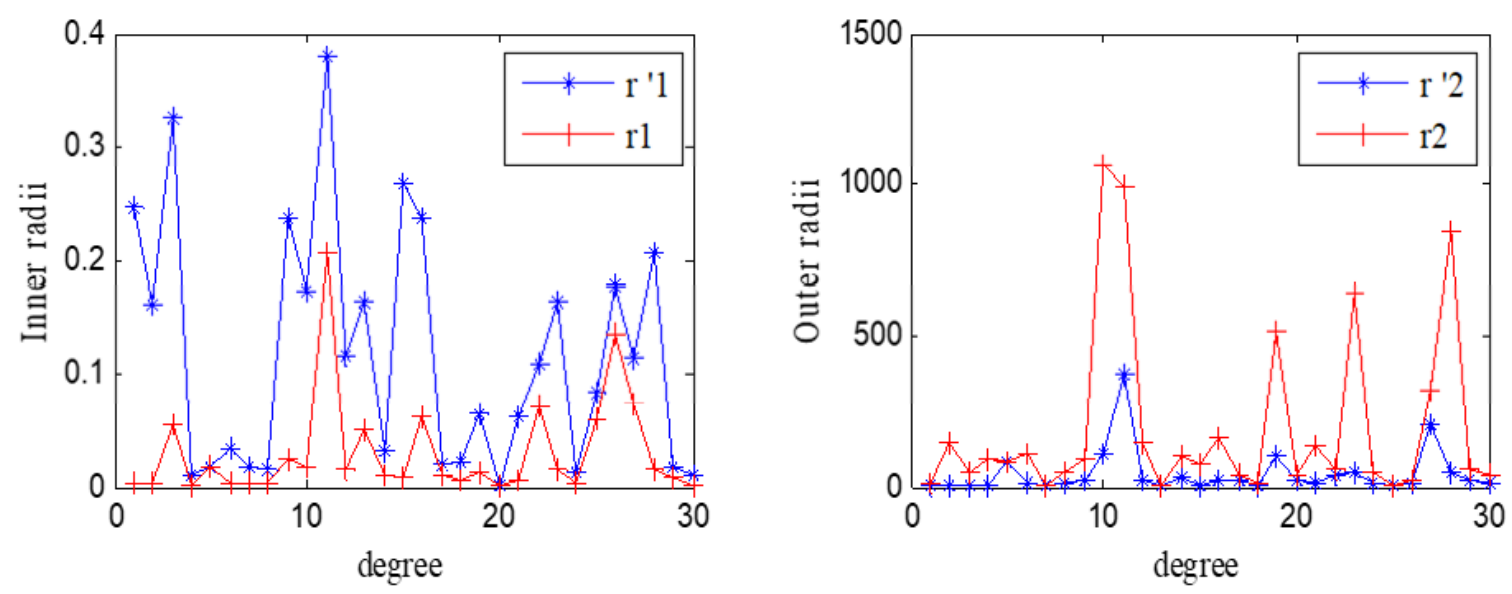

Figure 1. Comparison of bounds of Theorem 2.1 and Theorem 1.5

Therefore, we have

$$
\left\{\left|\frac{a_{0}}{a_{n}}\right|^{p} \frac{n^{p / q}}{r^{n p}}+\left|\frac{a_{1}}{a_{n}}\right|^{p} \frac{n^{p / q}}{r^{(n-1) p}}+\cdots+\left|\frac{a_{n-1}}{a_{n}}\right|^{p} \frac{n^{p / q}}{r^{p}}\right\}=1 .
$$

If we take

$$
\lambda_{k}=\left|\frac{a_{n-k}}{a_{n}}\right|^{p} \frac{n^{p / q}}{r^{k p}}, \quad k=1,2, \ldots, n,
$$

the equality (2.4) yields

$$
\sum_{k=1}^{n}\left|\lambda_{k}\right|=1
$$

Now, Theorem 2.1 implies that

$$
r_{2}=\max _{1 \leq k \leq n}\left\{\frac{n^{1 / q}}{\left|\lambda_{k}\right|^{1 / p}}\left|\frac{a_{n-k}}{a_{n}}\right|\right\}^{1 / k}=r .
$$

Hence, it follows that all the zeros of a polynomial $P(z)$ lie in $|z| \leq r$, where $r$ is the positive root of the equation (2.3). This generalizes Theorem 1.2 due to Cauchy [4], which is fairly deduced by our Theorem 2.1. The lower bound can be obtained in a similar way. Taking $q \rightarrow+\infty(p \rightarrow 1)$, this result reduces to Theorem 1.2.

Table 1 represents corollaries using some of the existing identities. The left column of the table contains the identities used in place of $\left|\lambda_{k}\right|$ in Theorem 2.1 and the right column shows the extension of corresponding existing results. So many other results can be obtained using different identities.

Remark 2.3. By (2.5) and (2.6), one can see that the best possible result this direction comes when $D_{r_{1}}^{\prime}:=1-\left(\sum_{k=1}^{n}\left|\frac{a_{k}}{a_{0}}\right|^{p} n^{p / q} r_{1}^{k p}\right)^{1 / p}=0$ and 
TABLE 1. Corollaries

\begin{tabular}{|l|l|}
\hline \multicolumn{1}{|c|}{$\left|\lambda_{k}\right|$} & \multicolumn{1}{|c|}{ Existing Results } \\
\hline 1. $\frac{2^{n-k} 3^{k} F_{k} C(n, k)}{F_{4 n}}, F_{k}$ is the $k^{\text {th }}$ Fibonacci number & Theorem 1.3 of $[9]$ \\
\hline 2. $\frac{C(n, k)}{2^{n}-1}, C(n, k)$ are the Binomial coefficients & Theorem 1.4 of $[12]$ \\
\hline 3. $\frac{C(n, k) A_{k} B_{j}^{k}\left(b B_{j-1}\right)^{n-k}}{A_{j n}}$ & Theorem 2 of [10] \\
\hline 4. $\frac{L_{k}}{L_{n+2}-3}, L_{k}$ is $k^{\text {th }}$ Lucas number & Corollary 2.1 of [5] \\
\hline 5. $\frac{C_{k-1} C_{n-k}}{C_{n}}, C_{k}$ is the $k^{\text {th }}$ Catalan number & Corollary 2.2 of [5] \\
\hline
\end{tabular}

$D_{r_{2}}^{\prime}:=1-\left(\sum_{k=1}^{n}\left|\frac{a_{n-k}}{a_{n}}\right|^{p} \frac{n^{p / q}}{r_{2}^{k p}}\right)^{1 / p}=0$. But, this result is implicit in the sense that in order to find the annulus containing all the zeros of a polynomial, we need to compute the roots of another associated equation (2.3). Our Theorem 2.1 provide a family of annuli that contains all the zeros of a polynomial based only on the polynomial coefficients.

Our next result improves upon any bound $r_{1}, r_{2}$ obtainable from any of the above mentioned results if $D_{r_{1}}^{\prime}>0$ and $D_{r_{2}}^{\prime}>0$.

Theorem 2.2. Let $\lambda_{1}, \lambda_{2}, \ldots, \lambda_{n}$ be any set of $n$ real or complex numbers such that $\sum_{k=1}^{n}\left|\lambda_{k}\right| \leq 1$. If $r_{1}$ and $r_{2}$ be any positive numbers such that $D_{r_{1}}^{\prime} \geq 0$ and $D_{r_{2}}^{\prime} \geq 0$, then for $p$ and $q$ such that $p>1, q>1, \frac{1}{p}+\frac{1}{q}=1$, the non-constant polynomial $P(z)=\sum_{k=0}^{n} a_{k} z^{k}, a_{k} \neq 0,1 \leq k \leq n$, has all its zeros lie in the annulus $\mathcal{C}=$ $\left\{z: r_{1}^{\prime \prime} \leq|z| \leq r_{2}^{\prime \prime}\right\}$, where

$$
r_{1}^{\prime \prime}=\min _{1 \leq k \leq n}\left(r_{1}^{k}+D_{r_{1}}^{\prime}\left|\frac{\lambda_{k}^{1 / p}}{n^{1 / q}} \frac{a_{0}}{a_{k}}\right|\right)^{1 / k}
$$

and

$$
r_{2}^{\prime \prime}=\max _{1 \leq k \leq n}\left(r_{2}^{-k}+D_{r_{2}}^{\prime}\left|\frac{\lambda_{k}^{1 / p}}{n^{1 / q}} \frac{a_{n}}{a_{n-k}}\right|\right)^{-1 / k} .
$$

Remark 2.4. (a) Since, it is clear that $r_{1}^{\prime \prime} \geq r_{1}$ and $r_{2}^{\prime \prime} \leq r_{2}$ therefore the Theorem 2.2 always gives bound sharper than any of the bound that has been obtained in terms of $r_{1}$ and $r_{2}$.

(b) In order to obtain better and better bound, Theorem 2.2 can be applied multiple times by substituting newly computed $r_{1}^{\prime \prime}$ and $r_{2}^{\prime \prime}$ as $r_{1}$ and $r_{2}$ in the formula, and making corresponding substitutions in $D_{r_{1}}^{\prime}$ and $D_{r_{2}}^{\prime}$ respectively and this has been shown in Section 4.

If we take $q \rightarrow+\infty(p \rightarrow 1)$ in the above Theorem 2.2 with $\lim _{q \rightarrow+\infty} D_{r_{1}}^{\prime}=$ $1-\sum_{k=1}^{n}\left|\frac{a_{k}}{a_{0}}\right| r_{1}^{k}=: D_{r_{1}}$ and $\lim _{q \rightarrow+\infty} D_{r_{2}}^{\prime}=1-\sum_{k=1}^{n}\left|\frac{a_{n-k}}{a_{n}}\right| \frac{1}{r_{2}^{k}}=: D_{r_{2}}$, we get the following corollary, which in particular gives a result due to Dalal and Govil [6]. 
Corollary 2.1. Let $\lambda_{1}, \lambda_{2}, \ldots, \lambda_{n}$ are any set of $n$ real or complex numbers such that $\sum_{k=1}^{m}\left|\lambda_{k}\right| \leq 1$. If $r_{1}$ and $r_{2}$ be any positive numbers such that $D_{r_{1}} \geq 0$ and $D_{r_{2}} \geq 0$, then the non-constant polynomial $P(z)=\sum_{k=0}^{n} a_{k} z^{k}, a_{k} \neq 0,1 \leq k \leq n$, has all its zeros lie in the annulus $\mathcal{C}=\left\{z: r_{1}^{\prime \prime} \leq|z| \leq r_{2}^{\prime \prime}\right\}$, where

$$
r_{1}^{\prime \prime}=\min _{1 \leq k \leq n}\left(r_{1}^{k}+D_{r_{1}}\left|\lambda_{k} \frac{a_{0}}{a_{k}}\right|\right)^{1 / k}
$$

and

$$
r_{2}^{\prime \prime}=\max _{1 \leq k \leq n}\left(r_{2}^{-k}+D_{r_{2}}\left|\lambda_{k} \frac{a_{n}}{a_{n-k}}\right|\right)^{-1 / k}
$$

\section{Proof of the Theorems}

Proof of Theorem 2.1. Proof of Theorem 2.1 follows on the same lines of Theorem 2.2, therefore we omit the details.

Proof of Theorem 2.2. If $a_{0}=0$, then $r_{1}^{\prime}=0$ which implies $r_{1}^{\prime \prime}=r_{1}^{\prime}=0$. Therefore, without loss of generality, we can suppose that $a_{0} \neq 0$. Let $z$ be such that $|z|<r_{1}^{\prime \prime}$. Then we have

$$
\begin{aligned}
|P(z)| & \geq\left|a_{0}\right|-\left|\sum_{k=1}^{n} a_{k} z^{k}\right| \geq\left|a_{0}\right|-\sum_{k=1}^{n}\left|a_{k}\right||z|^{k}>\left|a_{0}\right|-\sum_{k=1}^{n}\left|a_{k}\right|\left(r_{1}^{\prime \prime}\right)^{k} \\
& =\left|a_{0}\right|\left[1-\sum_{k=1}^{n}\left|\frac{a_{k}}{a_{0}}\right|\left(r_{1}^{\prime \prime}\right)^{k}\right] .
\end{aligned}
$$

Now, from (2.7), we get

$$
\left|\frac{a_{k}}{a_{0}}\right|\left(r_{1}^{\prime \prime}\right)^{k} \leq\left|\frac{a_{k}}{a_{0}}\right| r_{1}^{k}+D_{r_{1}}^{\prime} \frac{\left|\lambda_{k}\right|^{1 / p}}{n^{1 / q}}, \quad \text { for } k=1,2, \ldots, n .
$$

Substituting (3.2) in (3.1), and using Hölder inequality (1.3), we obtain

$$
\begin{aligned}
|P(z)| & >\left|a_{0}\right|\left[1-\sum_{k=1}^{n}\left|\frac{a_{k}}{a_{0}}\right| r_{1}^{k}-\sum_{k=1}^{n} D_{r_{1}}^{\prime} \frac{\left|\lambda_{k}\right|^{1 / p}}{n^{1 / q}}\right] \\
& \geq\left|a_{0}\right|\left[1-n^{1 / q}\left(\sum_{k=1}^{n}\left|\frac{a_{k}}{a_{0}}\right|^{p} r_{1}^{k p}\right)^{1 / p}-D_{r_{1}}^{\prime}\left(\sum_{k=1}^{n}\left|\lambda_{k}\right|\right)^{1 / p}\right] \\
& =\left|a_{0}\right|\left[D_{r_{1}}^{\prime}-D_{r_{1}}^{\prime}\left(\sum_{k=1}^{m}\left|\lambda_{k}\right|\right)^{1 / p}\right] \\
& =\left|a_{0}\right| D_{r_{1}}^{\prime}\left[1-\left(\sum_{k=1}^{m}\left|\lambda_{k}\right|\right)^{1 / p}\right] \geq 0
\end{aligned}
$$

as $\sum_{k=1}^{m}\left|\lambda_{k}\right| \leq 1$, and consequently $P(z)$ does not have any zero in $|z|<r_{1}^{\prime \prime}$. 
To prove the second part of the theorem, we shall use the first part. Consider the polynomial

$$
Q(z)=z^{n} P\left(\frac{1}{z}\right)=a_{0} z^{n}+a_{1} z^{n-1}+\cdots+a_{n-1} z+a_{n} .
$$

By the first part of the theorem, all the zeros of the polynomial $Q(z)$ lie in

$$
\begin{aligned}
|z| & \geq \min _{1 \leq k \leq n}\left(\frac{1}{r_{2}^{k}}+\frac{D_{r_{2}}^{\prime}\left|\lambda_{k}\right|^{1 / p}}{n^{1 / q}}\left|\frac{a_{n}}{a_{n-k}}\right|\right)^{1 / k} \\
& =\min _{1 \leq k \leq m} \frac{1}{\left(r_{2}^{-k}+\frac{D_{r_{2}}^{\prime}\left|\lambda_{k}\right|^{1 / p}}{n^{1 / q}}\left|\frac{a_{n}}{a_{n-k}}\right|\right)^{-1 / k}} \\
& =\frac{1}{\max _{1 \leq k \leq m}\left(r_{2}^{-k}+\frac{D_{r_{2}}^{\prime}\left|\lambda_{k}\right|^{1 / p}}{n^{1 / q}}\left|\frac{a_{n}}{a_{n-k}}\right|\right)^{-1 / k}}=\frac{1}{r_{2}^{\prime \prime}} .
\end{aligned}
$$

Replacing $z$ by $\frac{1}{z}$ and observing that $P(z)=z^{n} Q\left(\frac{1}{z}\right)$, we conclude that all the zeros of $P(z)$ lie in

$$
|z| \leq r_{2}^{\prime \prime}=\max _{1 \leq k \leq m}\left(r_{2}^{-k}+\frac{D_{r_{2}}^{\prime}\left|\lambda_{k}\right|^{1 / p}}{n^{1 / q}}\left|\frac{a_{n}}{a_{n-k}}\right|\right)^{-1 / k}
$$

and (2.8) is thus established.

\section{Numerical EXPERIMENTS}

By Figure 2.1, it is easy to see that for any random polynomial, random sequence of $\lambda_{k}, \sum_{k=0}^{n}\left|\lambda_{k}\right|<1 / n, 1 \leq k \leq n$ and random $p>1, q>1$, with $1 / p+1 / q=1$, our Theorem 2.1 always gives better bounds than Theorem 1.5. Here, we construct an example of a polynomial in order to compare our results, Theorems 2.1 and 2.2, with Theorem 1.5.

Example 4.1. Let $P(z)=z^{4}+0.01 z^{3}+0.1 z^{2}+0.2 z+0.4$.

TABLE 2. Comparison of Annuli (Theorem 2.2 applying again to $P(z)$ using the bounds $r_{1}=0.5187$ and $\left.r_{2}=1.0628\right)$

\begin{tabular}{lccc}
\hline Result & $r_{1}$ & $r_{2}$ & Area of annulus \\
\hline Theorem 1.5 & 0.2847 & 1.1647 & 4.0070 \\
Theorem 2.1 & 0.4343 & 1.1345 & 3.4509 \\
Theorem 2.2 & 0.5187 & 1.0628 & 2.7033 \\
Theorem 2.2 & 0.5539 & 1.0442 & 2.4615 \\
Actual bound & 0.7412 & 0.8532 & 0.5610 \\
\hline
\end{tabular}


In Table 2, we first apply Theorems 1.5 and 2.1 to $P(z)$ using the random sequence $\lambda_{k}, \sum_{k=0}^{n}\left|\lambda_{k}\right|<1 / n$ and random $p>1, q>1$, with $1 / p+1 / q=1$. We see that Theorem 2.1 gives sharper bounds than Theorem 1.5. We then apply Theorem 2.2 to $P(z)$ using the same sequence of $\lambda_{k}, p, q$ and the radii $r_{1}=0.4343$ and $r_{2}=1.1345$, and we obtain better bounds than Theorem 2.1. Applying again Theorem 2.2 to $P(z)$ using the radii $r_{1}=0.5187$ and $r_{2}=1.0628$ of Theorem 2.2 , we get new bounds $r_{1}^{\prime \prime}=0.5539$ and $r_{2}^{\prime \prime}=1.0442$, which are more closer to the actual radii of the annulus that contains all the zeros of $P(z)$.

Acknowledgements. The authors are extremely grateful to the referee for his valuable suggestions.

\section{REFERENCES}

[1] C. Affane-Aji, S. Biaz and N. K. Govil, On annuli containing all the zeros of a polynomial, Math. Comp. Modelling 52 (2010) 1532-1537.

[2] A. Aziz and A. Qayoom, Estimates for the modulii of the zeros of a polynomial, Math. Inequal. Appl. 9 (2006) 107-116.

[3] M. Bidkham and E. Shashahani, An annulus for the zeros of polynomials, Appl. Math. Lett. 24 (2011) 122-125.

[4] A. L. Cauchy, Exercises de Mathématiques, IV, Anné de Bure Fréres, Paris, 1829.

[5] A. Dalal and N. K. Govil, On region containing all the zeros of a polynomial, Appl. Math. Comput. 219 (2013) 9609-9614.

[6] A. Dalal and N. K. Govil, Annulus containing all the zeros of a polynomial, Appl. Math. Comput. 249 (2014) 429-435.

[7] A. Dalal and N. K. Govil, On comparison of annuli containing all the zeros of a polynomial, Appl. Anal. Discrete Math. 11 (2017) 232-241.

[8] B. Datt and N. K. Govil, On the location of zeros of a polynomial, J. Approx. Theory 24 (1978) 78-82.

[9] J. L. Diaz-Barrero, An annulus for the zeros of polynomials, J. Math. Anal. Appl. 273 (2002) 349-352.

[10] J. L. Diaz-Barrero and J. J. Egozcue, Bounds for the moduli of zeros, Appl. Math. Lett. 17 (2004) 993-996.

[11] A. Joyal, G. Labelle and Q. I. Rahman, On the location of zeros of polynomials, Canad. Math. Bull. 10 (1967) 53-63.

[12] S. H. Kim, On the moduli of the zeros of a polynomial, Amer. Math. Monthly 112 (2005) 924-925.

[13] M. Marden, Geometry of Polynomials, 2nd edition, Mathematical Surveys 3, Amer. Math. Soc. Providence, RI, USA, 1966.

[14] G. V. Milovanović, D. S. Mitrinović and Th. M. Rassias, Topics in Polynomials: Extremal Problems, Inequalities and Zeros, World Scientific, Singapore, 1994.

[15] Q. I. Rahman and G. Schmeisser, Analytic Theory of Polynomials, Oxford University Press Inc., New York, 2002.

[16] Y. J. Sun and J. G. Hsieh, A note on circular bound of polynomial zeros, IEEE Trans. Circuits Syst. I. Regul. Pap. 43 (1996) 476-478. 
${ }^{1}$ Department of Applied Mathematics,

Amity University,

NoIDA-201313, INDIA

Email address: sunil.hans82@yahoo.com

Email address: atomar@amity.edu

${ }^{2}$ School of Mathematics and Computing Science,

Guilin University of Electronic Technology,

Guilin-541004, ChinA

Email address: cjh868@126.com 